\title{
GRAPHS AND COMMON FIXED POINT THEOREMS FOR SEQUENCES OF MAPS
}

\author{
JACEK R. JACHYMSKI \\ Institute of Mathematics \\ Technical University \\ Żwirki 36, 90-924 Lódź \\ Poland
}

(Received April 26, 1995)

\begin{abstract}
We demonstrate a usefulness of the notion of a connected graph for obtaining some common fixed point theorems. In particular, we establish two theorems of this type involving one, two and four sequences of maps. This generalizes among others the recent results of $S$. Chang [1], J. Jachymski [2], S. Sessa, R. N. Mukherjee and T. Som [3], and T. Taniguchi [4].
\end{abstract}

KEY WORDS AND PHRASES. Common fixed point, contractive gauge function, compatible maps, connected graph.

1992 AMS SUBJECT CLASSIFICATION CODES. 47H10, 54H25.

\section{INTRODUCTION.}

There are a number of papers dealing with fixed points for sequences of maps. B. E. Rhoades [5] divided these results into four categories (for the details, see [5], p. 10). In this paper we extend the fourth class of theorems discussed in [5]. In this situation maps $A_{i}$ and $A_{j}$ or $A_{i}$ and $B_{j}$ satisfy pairwise a contractive type condition involving eventually other maps, with contractive gauge functions, which may depend on $i$ and $j$. To get a common fixed point theorem, most of the authors use an iteration procedure involving all of the maps considered (see, e.g., [3], [4], [6]). Then, however, the same contractive gauge function is employed, or some additional hypothesis on gauge functions are imposed (see, e.g., the comments in [5], p. 13-14).

Recently, G. Jungck et al. [7] and B. E. Rhoades [5] obtained common fixed point theorems for a sequence of maps using earlier results involving a finite number of maps. As has been pointed out in [5], such a way of treatment enables one to use different contractive gauge functions, without any additional hypotheses (see Corollary 1 [5]). A theorem of this type has been also established in the recent article [2].

In this paper we give a precise description of a set of positive integers $(i, j)$ for which a contractive condition is to be satisfied in order to guarantee the existence of a common fixed point. Most of the authors assume that a contractive condition is to hold for all $(i, j)$ with $i \neq j$ 
(see, e.g., [1], [3], [7], [8]). However, H. Chatterji has observed that it suffices to use a contractive conditions for pairs

$$
(i, j) \in J:=\{(1, n+1): n \in \mathbf{N}\}
$$

only ( $\mathbf{N}$ denotes the set of all positive integers). The same set $J$ is employed in Theorem 5.1 [2]. All of the theorems mentioned here deal with a single sequence of maps. On the other hand, $T$. Taniguchi [4] establishing a fixed point theorem for two sequences of maps, has assumed that a contractive condition holds for all pairs

$$
\left.(i, j) \in J_{T}:=\{(2 n-1,2 n): n \in \mathbf{N}\} \cup\{2 n, 2 m+1): m \geq n \geq 1\right\}
$$

In the next section we show that, thanks to the notion of a connected graph, it is possible to unify and extend all of the above results. Moreover, our conditions imposed on a set $J$ involving a graph appear to be necessary and sufficient for the existence of a common fixed point (see Theorems 2.2 and 2.3).

\section{A FIXED POINT THEOREM INVOLVING GRAPH.}

We start by recalling a common fixed point theorem for four maps ([2], Theorem 3.3). It can be deduced from Lemma 1 [9], that though this theorem involves a contractive gauge function, it yields the recent result of Jungck et al. [7] involving $(\epsilon, \delta)$-type conditions. For the definition of compatible maps, a generalization of the commutative map concept, see [10]. The letter $\mathbf{R}_{+}$ denotes the set of all nonegative reals.

THEOREM 2.1. Let $A, B, S$ and $T$ be selfmaps of a complete metric space $(X, d)$, and let $\Phi: \mathbf{R}_{+} \mapsto \mathbf{R}_{+}$be an upper semicontinuous (not necessarily monotonic) function such that $\Phi(t)<t$ for $t>0$. Let $(A, B, S, T)$ satisfy the following conditions:

$$
\begin{gathered}
d(A x, B y) \leq \Phi(\max \{d(S x, T y), d(A x, S x), d(B y, T y), \\
\left.\left.\frac{1}{2}[d(A x, T y)+d(B y, S x)]\right\}\right), \text { for all } x, y \in X \\
A(X) \subseteq T(X) \text { and } B(X) \subseteq S(X)
\end{gathered}
$$

the pairs $A, S$ and $B, T$ are compatible;

one of $A, B, S$ and $T$ is continuous.

Then $A, B, S$ and $T$ have a unique common fixed point.

Further, let us recall that an undirected graph is a pair $\langle V, E\rangle$, where $V$ is a set and $E$ is a family of two-element subsets of $V$. A graph $\langle V, E\rangle$ is said to be connected if for each $x, y \in V$, there exists a finite sequence $\left\{x_{i}\right\}_{i=0}^{n}$ such that $x_{0}=x, x_{n}=y$ and $\left\{x_{i-1}, x_{i}\right\} \in E$, for $i=1, \ldots, n$ (see, e.g., [11]). The letters $\Pi_{1}$ and $\Pi_{2}$ denote the projections of $\mathrm{N}^{2}$ onto $\mathrm{N}$, i.e.,

$$
\Pi_{1}(n, m):=n, \text { and } \Pi_{2}(n, m):=m, \text { for }(n, m) \in \mathbf{N}^{2} .
$$


THEOREM 2.2. Let $A, B, S, T$ and $A_{n}, B_{n}, S_{n}(n \in \mathrm{N})$ be selfmaps of a complete metric space $(X, d)$. Let $J \subseteq N^{2}$ and for $(i, j) \in J, \Phi_{i j}: \mathbf{R}_{+} \mapsto \mathbf{R}_{+}$be an upper semicontinuous function such that $\Phi_{i j}(t)<t$ for $t>0$. Assume that one of the following conditions hold.

For each $(i, j) \in J,\left(A, B, S_{i}, S_{j}\right)$ satisfies $(2.1)$ with $\Phi:=\Phi_{i j}$,

$A(X) \cup B(X) \subseteq \bigcap_{n=1}^{\infty} S_{n}(X),\left(A, S_{n}\right)$ and $\left(B, S_{n}\right)$ are

compatible $(n \in \mathrm{N})$, and all the $S_{n}$ are continuous.

For each $(i, j) \in J,\left(A_{i}, A_{j}, S, T\right)$ satisfies (2.1) with $\Phi:=\Phi_{i j}$,

$A_{n}(X) \subseteq S(X) \cap T(X)$ for $n \in \mathrm{N},\left(A_{n}, S\right)$ and $\left(B, S_{n}\right)$ are

compatible $(n \in \mathrm{N})$, and $S$ or $T$ is continuous.

For each $(i, j) \in J,\left(A_{i}, A_{j}, B_{i}, B_{j}\right)$ satisfies (2.1) with $\Phi:=\Phi_{i j}$, (2.2), (2.3), and all the $B_{n}$ are continuous.

For each $(i, j) \in J,\left(A_{i}, B_{j}, B_{i}, A_{j}\right)$ satisfies (2.1) with $\Phi:=\Phi_{i j}$,

(2.2), $\left(A_{n}, B_{n}\right)$ are compatible $(n \in N)$, and for each $n \in N$,

$A_{n}$ or $B_{n}$ is continuous.

For each $(i, j) \in J,\left(A_{i}, B, S, A_{j}\right)$ satisfies (2.1) with $\Phi:=\Phi_{i j}$,

(2.2), (2.3), and $B$ or $S$ is continuous.

Further, define the family $E_{J}$ of two-element subsets of $J$ by

$$
\{(i, j),(k, l)\} \in E_{J} \quad \text { iff }(i, j),(k, l) \in J \text { and }\{i, j\} \cap\{k, l\} \neq \emptyset .
$$

If the graph $\left\langle J, E_{J}\right\rangle$ is connected and $\Pi_{1}(J) \cup \Pi_{2}(J)=\mathbf{N}$ then the following families of maps have a unique common fixed point.

(1) All the $S_{n}(n \in N), A$ and $B$ if (2.5) holds.

(2) All the $A_{n}(n \in \mathrm{N}), S$ and $T$ if (2.6) holds.

(3) All the $A_{n}$ and $B_{n}(n \in N)$ if (2.7) or (2.8) holds.

(4) All the $A_{n}(n \in N), B$ and $S$ if (2.9) holds.

PROOF. Assume that one of conditions (2.5)-(2.9) holds. By Theorem 2.1, for each $(i, j) \in J$ there is a unique common fixed point $z_{i j}$ for the suitable quaternion of maps. Let $(i, j),(k, l) \in J$ and $\{i, j\} \cap\{k, l\} \neq \emptyset$. Assume that $j=k$ or $j=l$. Then, by putting in (2.1), $\Phi:=\Phi_{i j}$, $x:=z_{i j}$ and $y:=z_{k l}$, and replacing $(A, B, S, T)$ by the suitable quaternion of maps, we obtain that $d(x, y) \leq \Phi_{i j}(d(x, y))$. Hence, $x=y$ since $\Phi_{i j}(t)<t$ for $t>0$. Assume that $i=k$ or $i=l$. Similarly, by putting in (2.1), $x:=z_{k l}$ and $y:=z_{i j}$ we obtain that $d(x, y) \leq \Phi_{i j}(d(x, y))$. Therefore, $z_{i j}=z_{k l}$.

Now, let $(i, j)$ and $(k, l)$ be arbitrary elements of $J$. By the connectivity of $\left\langle J, E_{J}\right\rangle$, there exists an $n \in \mathbf{N}$ and a sequence $\left\{\left(i_{k}, j_{k}\right)\right\}_{k=0}^{n}$ in $J$ such that $\left(i_{0}, j_{0}\right)=(i, j),\left(i_{n}, j_{n}\right)=(k, l)$, and $\left\{i_{k-1}, j_{k-1}\right\} \cap\left\{i_{k}, j_{k}\right\} \neq \emptyset$, for $k=1, \ldots, n$. Then, by the preceding part of the proof, $z_{i_{k-1} j_{k-1}}=z_{i_{k} j_{k}}$ for $k=1, \ldots, n$, which immediately gives $z_{i j}=z_{k l}$. This means, there is a $z_{0} \in X$ such that $z_{i j}=z_{0}$, for all $(i, j) \in J$. 
Now, fix an $n \in \mathbf{N}$ and assume that (2.5) holds. By hypothesis, there is a $k \in \mathbf{N}$ such that $(k, n) \in J$ or $(n, k) \in J$. For example, assume $(k, n) \in J$. Then $z_{k n}$ is the common fixed point of $A, B, S_{k}$ and $S_{n}$; in particular, $z_{0}\left(=z_{k n}\right)$ is the common fixed point of $A, B$ and $S_{n}$. The same argument may be used in the cases, in which any one of conditions (2.6)-(2.9) holds instead of (2.5).

REMARK 2.1. It is easy to verify that, for each of the sets $J_{k}(k=1,2,3,4)$ defined below, the graph $<J_{k}, E_{J_{k}}>$ is connected and $\Pi_{1}\left(J_{k}\right) \cup \Pi_{2}\left(J_{k}\right)=\mathrm{N}$.

(1) $J_{1}:=\{(i, j): i, j \in \mathbf{N}$ and $i \neq j\}$.

(2) $J_{2}:=\{(n, n+1): n \in \mathbb{N}\}$.

(3) $J_{3}:=\{(1, n+1): n \in \mathbf{N}\}$.

(4) $J_{4}:=\{(1,2 n): n \in \mathbf{N}\} \cup\{(2 n, 2 n+1): n \in \mathbf{N}\}$.

REMARK 2.2. By putting $J:=J_{1}$ and assigning the $\Phi_{i j}$ to be the same function, and assuming that (2.5) or (2.6) holds, Theorem 2.2 yields Theorem 2 of Chang [1] and Theorem 3 of Sessa et al. [3], respectively. Clearly, we may also put here $J:=J_{k}$ for any $k \in\{2,3,4\}$ in order to obtain the essential extensions of the above theorems.

REMARK 2.3. By putting in Theorem 2.2, $J:=J_{2}$ and

$$
\Phi_{i j}:=k t \text { for some } k \in(0,1), t \in \mathbf{R}_{+} \text {and }(i, j) \in J,
$$

and assuming that (2.7) holds, we obtain the result generalizing Theorem A of Taniguchi [4] who has employed the condition

$$
d\left(A_{i} x, A_{j} y\right) \leq k d\left(B_{i} x, B_{j} y\right), \text { for } x, y \in X \text { and }(i, j) \in J_{T}
$$

( $J_{T}$ is defined by (1.1)), which is less general than (2.1).

REMARK 2.4. By putting in Theorem 2.2, $J:=J_{3}$ and assuming that (2.6) holds, we obtain Theorem 5.1 of Jachymski [2], the generalization of an earlier result due to Chatterji [6].

REMARK 2.5. Clearly, conditions (2.5)-(2.9) may be weakened. It suffices to have the suitable quaternions of maps satisfy the assumptions of Theorem 2.1. We have slightly strengthened them for aesthetic reasons.

\section{A CONVERSE TO THEOREM 2.2 .}

The following theorem is a converse to Theorem 2.2. It appears that the connectivity of the graph $\left\langle J, E_{J}>\right.$ and the condition $\Pi_{1}(J) \cup \Pi_{2}(J)=N$ are necessary for the existence of a common fixed point. More precisely, we have.

THEOREM 3.1. Let $J \subseteq \mathrm{N}^{2}$ and $E_{J}$ be defined as in Theorem 2.2. If the graph $<J, E_{J}>$ is not connected or $\Pi_{1}(J) \cup \Pi_{2}(J) \neq N$, then there exist a complete metric space $(X, d)$ and selfmaps $S, T, A_{n}(n \in \mathbb{N})$ of $X$ for which (2.6) is satisfied with $\Phi_{i j}$ being the same linear function, and there is no common fixed point for the family of maps.

PROOF. Assume that $\Pi_{1}(J) \cup \Pi_{2}(J) \neq \mathbf{N}$. Then there exists an $n_{0} \in \mathbf{N}$ such that, for all $k \in \mathbf{N},\left(k, n_{0}\right) \notin J$ and $\left(n_{0}, k\right) \notin J$. Let $X:=\mathbf{R}_{+}$be endowed with the Euclidean metric, $S:=I$, the identity on $X, T:=S$,

$$
A_{n} x:=\frac{1}{2} x \text { for } n \neq n_{0} \text { and } x \in X, A_{n_{0}} x:=x+1 \quad(x \in X),
$$


and $\Phi_{i j}(t):=\frac{1}{2} t$ for $(i, j) \in J$ and $t \in \mathbf{R}_{+}$. Then, for all $i, j \neq n_{0},\left(A_{i}, A_{j}, S, T\right)$ satisfies (2.1). In particular, by the definition of $n_{0},(2.1)$ holds for each $\left(A_{i}, A_{j}, S, T\right)$ with $(i, j) \in J$. So it is clear that (2.6) is satisfied. But since $A_{n_{0}}$ is a fixed point free map, there is no common fixed point for the family considered.

Now assume that $\Pi_{1}(J) \cup \Pi_{2}(J)=\mathbf{N}$ and that the graph $<J, E_{J}>$ is not connected. Then $<J, E_{J}>$ is the sum of its connected components, i.e., $J=\cup_{n=1}^{p} J_{n}$, where $p \in \mathbf{N} \cup\{\infty\}, p \geq 2$, $J_{n} \cap J_{m}=\emptyset$ if $n \neq m$, and for each of the $n$ considered, $\left\langle J_{n}, E_{J_{n}}\right\rangle$ is connected. Hence and by the definition of $E_{J}$, if $n \neq m$ and $(i, j) \in J_{n},(k, l) \in J_{m}$ then $\{i, j\} \cap\{k, l\}=\emptyset$. Therefore, we infer that

$$
\left(\Pi_{1}\left(J_{n}\right) \cup \Pi_{2}\left(J_{n}\right)\right) \cap\left(\Pi_{1}\left(J_{m}\right) \cup \Pi_{2}\left(J_{m}\right)\right)=\emptyset \text {, for } n \neq m .
$$

Let $(X, d), S, T$ and $\Phi_{i j}((i, j) \in J)$ be defined as above. Let

$$
A_{n} x:=\frac{1}{2} x \text { for } x \in X \text { and } n \in \Pi_{1}\left(J_{1}\right) \cup \Pi_{2}\left(J_{1}\right),
$$

and $A_{n} x:=\frac{1}{2} x+\frac{1}{2}$ for $x \in X$ and the rest of $n$. If $(i, j) \in J_{1}$, then $A_{i} x=A_{j} x=\frac{1}{2} x(x \in X)$. If $(i, j) \in J-J_{1}$ then, for some $n \geq 2,(i, j) \in J_{n}$ so $i, j \in \Pi_{1}\left(J_{n}\right) \cup \Pi_{2}\left(J_{n}\right)$. Hence and by (3.1), $i, j \notin \Pi_{1}\left(J_{1}\right) \cup \Pi_{2}\left(J_{1}\right)$. Then, by the definition of $\left\{A_{n}\right\}_{n=1}^{\infty}$, we get that $A_{i} x=A_{j} x=\frac{1}{2} x+\frac{1}{2}(x \in X)$. Therefore, we may infer that (2.1) holds for each $\left(A_{i}, A_{j}, S, T\right)$ with $(i, j) \in J$. Consequently, (2.6) is satisfied. Simultaneously, the above family of maps has no common fixed point.

REMARK 3.1. It is easy to verify that for each of the sets $J_{2}, J_{3}$ and $J_{4}$ defined in Remark 2.1, the following condition holds:

"if for some $k \in\{2,3,4\}, J^{*} \subseteq J_{k}$ and $J^{*} \neq J_{k}$, then either $\Pi_{1}\left(J^{*}\right) \cup \Pi_{2}\left(J^{*}\right) \neq N$, or the graph $<J^{*}, E_{J^{*}}>$ is not connected".

In view of Theorem 3.1, we may infer that $J_{2}, J_{3}$ and $J_{4}$ are minimal subsets of $\mathrm{N}^{2}$, which may be used in Theorem 2.2. Obviously, there are a number of another examples of such minimal sets.

\section{COMMON FIXED POINTS FOR FOUR SEQUENCES OF MAPS.}

Finally, using again the concept of a connected graph, we establish a common fixed point theorem for four sequences of maps.

THEOREM 4.1. Let $\left\{A_{n}\right\}_{n=1}^{\infty},\left\{B_{n}\right\}_{n=1}^{\infty},\left\{S_{n}\right\}_{n=1}^{\infty}$ and $\left\{T_{n}\right\}_{n=1}^{\infty}$ be sequences of selfmaps of a complete metric space $(X, d)$. Let $J \subseteq \mathbf{N}^{2}$ and for $(i, j) \in J, \Phi_{i j}: \mathbf{R}_{+} \mapsto \mathbf{R}_{+}$be an upper semicontinuous function such that $\Phi_{i j}(t)<t$ for $t>0$. Assume that, for each $(i, j) \in J$, $\left(A_{i}, B_{j}, S_{i}, T_{j}\right)$ satisfies conditions (2.1)-(2.4) with $\Phi:=\Phi_{i j}$. Further, define the family $F_{J}$ of two-element subsets of $J$ by

$$
\{(i, j),(k, l)\} \in F_{J} \quad \text { iff }(i, j),(k, l) \in J \text { and } i=k \text { or } j=l .
$$

If the graph $\left(J, F_{J}\right)$ is connected and $\Pi_{1}(J)=\Pi_{2}(J)=\mathbf{N}$, then all the $A_{n}, B_{n}, S_{n}$ and $T_{n}$ $(n \in \mathbf{N})$ have a unique common fixed point. 
PROOF. By Theorem 2.1, for each $(i, j) \in J$ there is a common fixed point $z_{i j}$ of maps $A_{i}, B_{j}, S_{i}$ and $T_{j}$. Let $(i, j),(k, l) \in J$ and $\{(i, j),(k, l)\} \in F_{J}$. Let $A:=A_{i}, B:=B_{j}, S:=$ $S_{i}, T:=T_{j}$ and $\Phi:=\Phi_{i j}$. By the definition of $F_{J}$, we have that $i=k$ or $j=l$. In the first case,

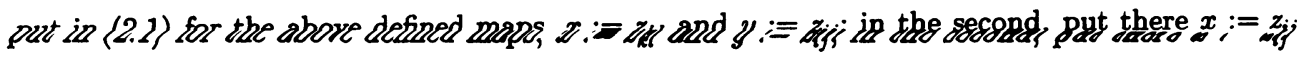
and $y:=z_{k l}$. In both cases we obtain that $d(x, y) \leq \Phi_{i j}(d(x, y))$. Hence, $x=y$, i.e. $z_{i j}=z_{k l}$ since $\Phi_{i j}(t)<t$ for $t>0$.

Now, if $(i, j)$ and $(k, l)$ are arbitrary elements of $J$, we may obtain, employing the connectivity of $\left\langle J, F_{J}\right\rangle$ and using the same argument as in the proof of Theorem 2.2, that $z_{i j}=z_{k l}$. Thus, there is a $z_{0} \in X$ such that $z_{i j}=z_{0}$, for all $(i, j) \in J$. Now, let $n \in \mathbf{N}$. By hypothesis, there exist $i, j \in \mathbf{N}$ such that $(i, n),(n, j) \in J$. Then $z_{i n}$ is a fixed point of $B_{n}$ and $T_{n}$, and $z_{n j}$ is a fixed point of $A_{n}$ and $S_{n}$. Since $z_{i n}=z_{n j}=z_{0}, z_{0}$ is the common fixed point of $A_{n}, B_{n}, S_{n}$ and $T_{n}$.

REMARK 4.1. It is easy to verify that for each of the sets $J_{k}(k=1,2,3)$ defined below, the graph $<J_{k}, F_{J_{k}}>$ is connected and $\Pi_{1}\left(J_{k}\right)=\Pi_{2}\left(J_{k}\right)=\mathrm{N}$.

(1) $J_{1}:=\{(i, j): i, j \in \mathbf{N}$ and $i \neq j\}$.

(2) $J_{2}:=\{(n, n+1): n \in \mathbf{N}\} \cup\{(n, n+2): n \in \mathbf{N}\} \cup\left\{\left(n_{0}+1,1\right)\right\}$, where $n_{0} \in \mathbf{N}$ is fixed.

(3) $J_{3}:=\{(1, n+1): n \in \mathbf{N}\} \cup\{(n, n+1): n \in \mathbf{N}\} \cup\left\{\left(n_{0}+1,1\right)\right\}$, where $n_{0} \in \mathbf{N}$ is fixed.

Moreover, $J_{2}$ and $J_{3}$ are minimal subsets of $\mathrm{N}^{2}$, which may be used in Theorem 4.1.

ACKNOWLEDGEMENT. The author is grateful to the referee for editiorial improvement of this paper.

\section{REFERENCES}

1. CHANG, S. On Common Fixed Point Theorem for a Family of $\Phi$ - contraction mappings, Math. Japon. 29 (1984), 527-536.

2. JACHYMSKI, J. R. Common Fixed Point Theorems for Some Families of Maps, Indian J. Pure Appl. Math. 25 (1994), 925-937.

3. SESSA, S., MUKHERJEE, R. N. and SOM, T. A Common Fixed Point Theorem for Weakly Commuting Mappings, Math. Japon. 31 (1986), 235-245.

4. TANIGUCHI, T. A Common Fixed Point Theorem for Two Sequences of Self-mappings,

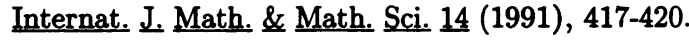

5. RHOADES, B. E. Fixed Point Theorems for Some Families of Maps, Indian J. Pure Appl. Math. 21 (1990), 10-20.

6. CHATTERJI, H. A Note on Common Fixed Points and Sequences of Mappings, Bull. Calcutta Math. Soc. 72 (1980), 139-142.

7. JUNGCK, G., MOON, K. P., PARK, S. and RHOADES, B. E. On Generalizations of the Meir-Keeler Type Contraction Maps: Corrections, J. Math. Anal. Appl. (1993).

8. HADŽIC, O. Common Fixed Point Theorems for Family of Mappings in Complete Metric Spaces, Math. Japon. 29 (1984), 127-134.

9. JACHYMSKI, J. R. Equivalent Conditions and the Meir-Keeler Type Theorems, J. Math. Anal. Appl. (to appear).

10. JUNGCK, G. Compatible Mappings and Common Fixed Points, Internat. J. Math. \& Math. Sci. $\underline{9}$ (1986), 771-779.

11. ÖRE, O. Theory of Graphs, Amer. Math. Soc. Coll. Publ. 38, Providence, R. I., 1962. 


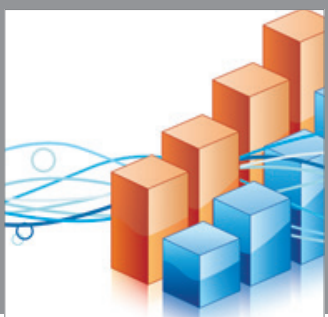

Advances in

Operations Research

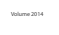

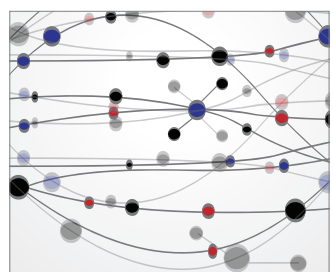

\section{The Scientific} World Journal
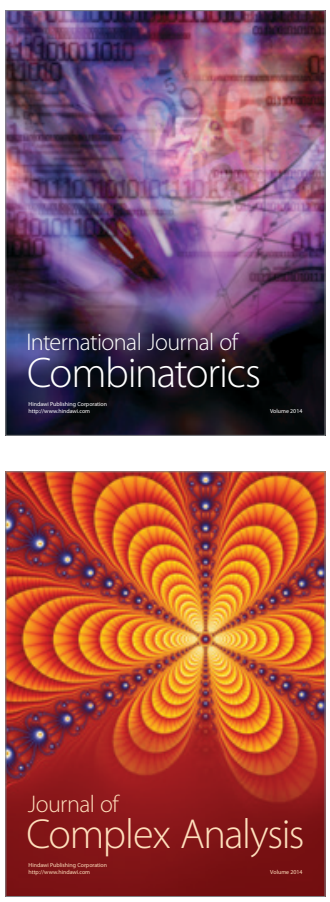

International Journal of

Mathematics and

Mathematical

Sciences
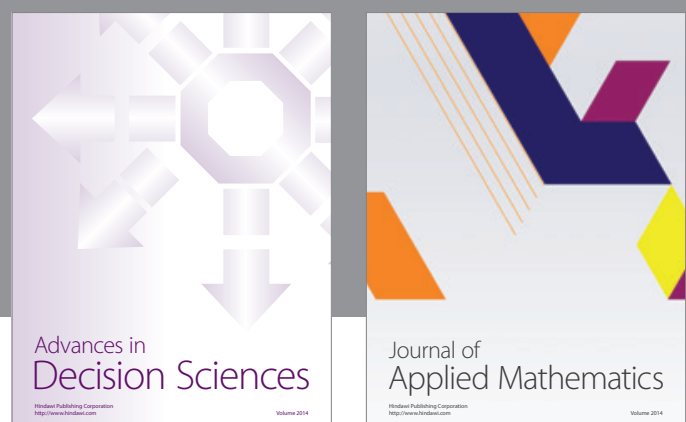

Journal of

Applied Mathematics
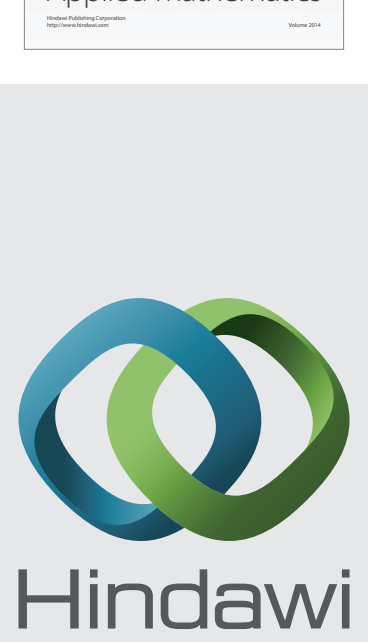

Submit your manuscripts at http://www.hindawi.com
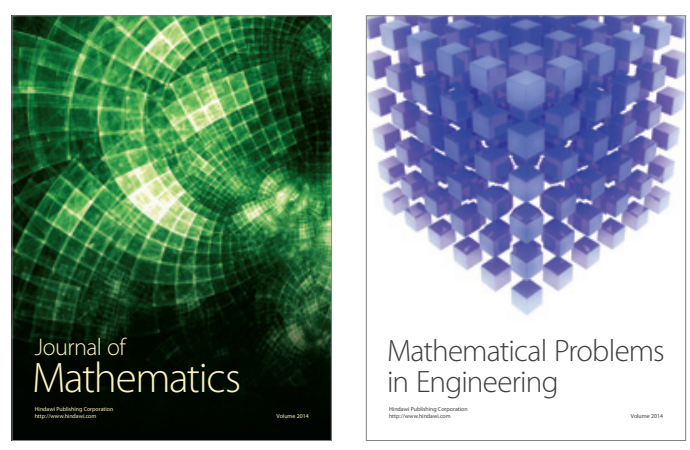

Mathematical Problems in Engineering
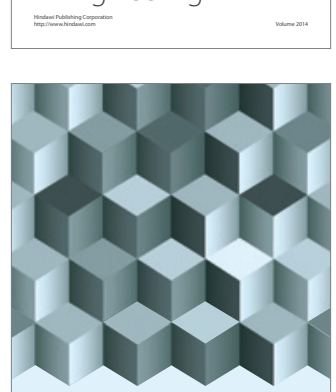

Journal of

Function Spaces
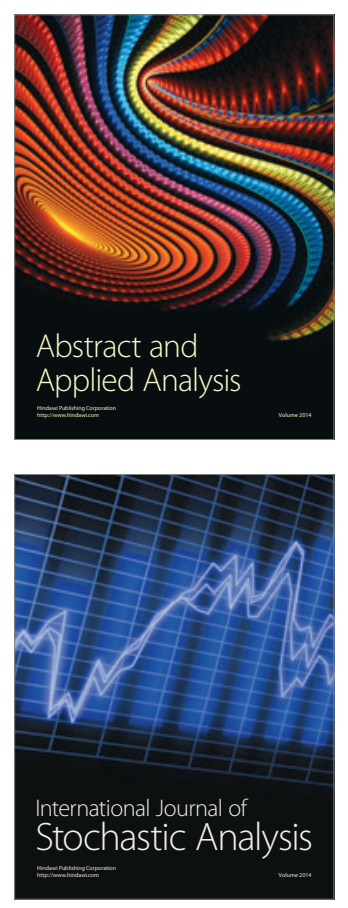

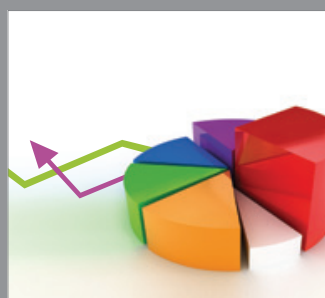

ournal of

Probability and Statistics

Promensencen
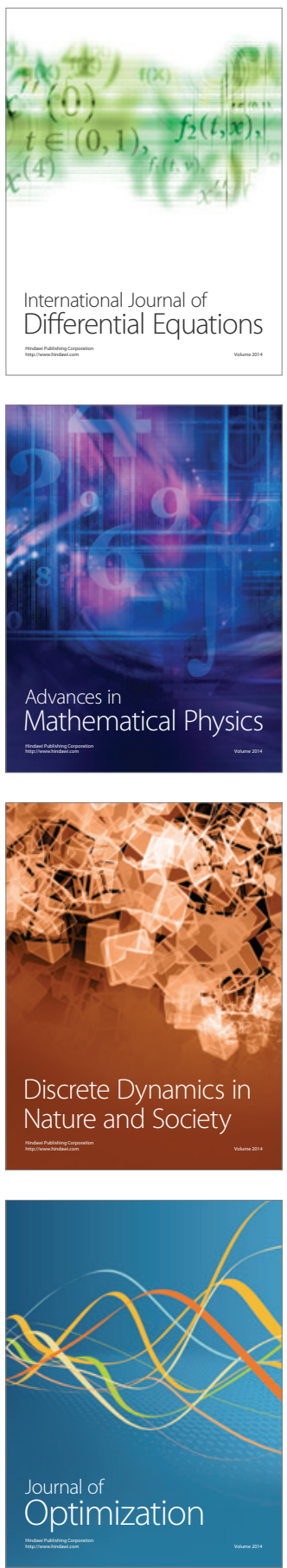